
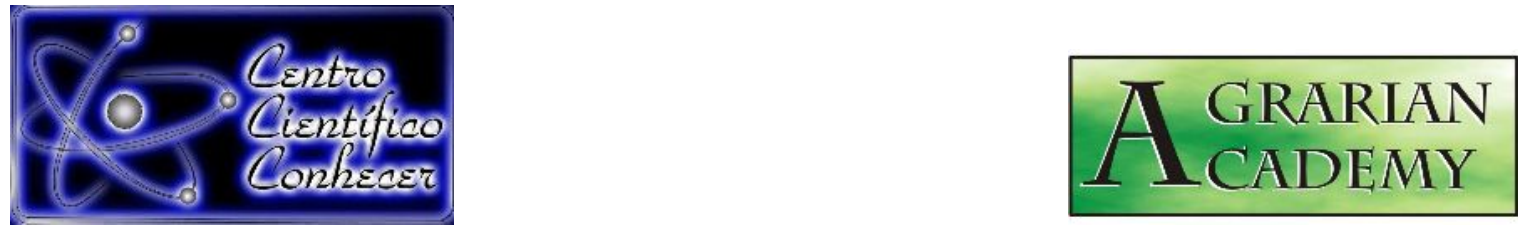

\title{
EQUAÇÕES PARA VARIÁVEIS DENDROMÉTRICAS EM ÁRVORES SINGULARES DE Pinus taeda L.
}

Emanuel Arnoni Costa ${ }^{1}$; Mário Cunha Sequeira ${ }^{2}$; Gabriel Paes Marangon ${ }^{3}$; Matheus Teixeira Martins ${ }^{4}$; Cristine Tagliapietra Schons ${ }^{5}$

${ }^{1}$ Prof. Dr. do Curso de Engenharia Florestal, Universidade Federal de Uberlândia, MG, Brasil (emanuelarnonicost@hotmail.com)

${ }^{2}$ Aluno do Curso de Engenharia Florestal, Universidade Federal de Uberlândia, MG, Brasil

${ }^{3}$ Prof. Dr. do Curso de Engenharia Florestal, Universidade Federal do Pampa, RS, Brasil

${ }^{4}$ Aluno do Curso de Engenharia Florestal, Universidade Federal do Pampa, RS, Brasil

${ }^{5}$ Doutoranda do Programa de Pós-Graduação em Engenharia Florestal, Universidade Federal de Santa Maria, RS, Brasil

Recebido em: 02/06/2019 - Aprovado em: 15/06/2019 - Publicado em: 22/07/2019 DOI: 10.18677/Agrarian_Academy_2019a15

\section{RESUMO}

Equações de variáveis dendrométricas foram desenvolvidas em árvores singulares de Pinus taeda L. em Capão Alto, SC. As árvores amostradas são provenientes de áreas de campo aberto. As árvores foram abatidas e submetidas a cubagem rigorosa do tronco. A técnica de Modelos Lineares Generalizados (MLGs) foi usada para modelar as variáveis de diâmetro à altura do peito (DAP), altura (h) e volume (v) em função do diâmetro medido a $0,1\left(\mathrm{~d}_{0,1}\right)$ e $0,3\left(\mathrm{~d}_{0,3}\right)$ metros acima do nível do solo. Os resultados indicam que é possível estimar com acurácia e precisão as variáveis dendrométricas em função do $\mathrm{d}_{0,3}$. As equações ajustadas podem auxiliar na quantificação dos atributos da árvore com base nas dimensões do toco.

PALAVRAS-CHAVE: modelos estatísticos, mensuração florestal, variáveis biométricas.

\section{EQUATIONS OF DENDROMETRIC VARIABLES IN SINGLE TREES OF PinUS taeda L.}

\section{ABSTRACT}

Dendrometric variables equations were developed on single trees of Pinus taeda $\mathrm{L}$. in Capão Alto, SC. The trees sampled come from open field areas. The trees were felled and subjected to rigorous cubing of the trunk. The Generalized Linear Models (GLMs) technique was used to model the variables of diameter at breast height AGRARIAN ACADEMY, Centro Científico Conhecer - Goiânia, v.6, n.11; p. 155 
$(\mathrm{DBH})$, height $(\mathrm{h})$ and volume $(\mathrm{v})$ as a function of diameter measured at $0.1\left(\mathrm{~d}_{0.1}\right)$ and $0.3\left(\mathrm{~d}_{0.3}\right)$ meters above ground level. The results indicate that it is possible to precision and accurately estimate the dendrometric variables as a function of $\mathrm{d}_{0.1}$ and $\mathrm{d}_{0.3}$. The fitted equations can aid in the quantification of the attributes of the tree based on the dimensions of the stump.

KEYWORDS: statistical models, forest measurement, biometric variables.

\section{INTRODUÇÃO}

O Brasil, devido a sua grande extensão territorial e sua condição climática, atualmente é uma referência internacional na produção de monoculturas voltadas para o setor florestal. As florestas plantadas no Brasil, se estendem por cerca de 7,84 milhões de hectares, em sua grande maioria composta por eucalipto e pinus (IBÁ, 2017). Grande parte dessa produção é destinada para a indústria de papel e celulose, já uma porção menor é destinada para produzir carvão vegetal, madeira serrada e borracha.

Segundo o IBGE (2017), no país, 95,8\% das áreas de florestas plantadas para fins comerciais são dedicadas ao cultivo de eucalipto e pinus, sendo $75,2 \%$ de eucalipto e $20,6 \%$ de pinus. O líder entre os estados é o Paraná, com $R \$ 3,7$ bilhões de valor de produção, seguido por Minas Gerais, com R\$3,3 bilhões, e Santa Catarina, com $R \$ 1,8$ bilhão. Do total de áreas plantadas, 41,9\% do eucalipto estão na Região Sudeste e $87,7 \%$ do pinus ficam na Região Sul.

A produção expressiva de pinus, especialmente no sul do país, está diretamente ligada ao aperfeiçoamento de técnicas e ao avanço de estudos, com propósito de otimizar os processos produtivos, tendo em vista o aspecto ambiental, econômico e social. Nesse contexto, é importante conhecer e estudar o desenvolvimento florestal para fins comerciais, quantificando a produção por meio de modelos estatísticos para auxiliar as atividades de planejamento florestal (IBÁ, 2017).

O diâmetro à altura do peito (DAP) é uma das variáveis da árvore mais importantes na silvicultura, sendo utilizado para descrever a estrutura, estimar o volume e selecionar árvores amostras em inventários (RIVAS et al., 2007). Desta forma, quando uma árvore é cortada e apenas o toco permanece como um indicador de seu tamanho, faz-se necessário utilizar as dimensões de toco para predizer o diâmetro à altura do peito e/ou o volume da árvore, verificar as práticas de colheita após a remoção das árvores, determinar a perda de volume resultante de cortes ilícitos e/ou avaliar os danos causados por condições ambientais (ARANDA et al., 2003).

Peter e Constance (2010) relatam que após um distúrbio em uma floresta é possível identificar informações históricas que podem indicar suas características estruturais e sua composição antes deste, bem como a sua reconstituição, suprindo, por exemplo, a falta de registro de informações dendrométricas, especialmente em cortes ilegais. Neste sentido, a reconstituição da dimensão de indivíduos lenhosos removidos a partir dos tocos deixados no solo, é uma prática comum, a partir da alternativa de avaliação de indivíduos em áreas vizinhas e a associação entre a dimensão dos tocos e o porte dos indivíduos (ERCANLI et al., 2015).

Quando um povoamento passa por um distúrbio, como por exemplo cortes rasos, também existe a possibilidade de se reconstituir a biomassa e/ou volume a partir dos diâmetros à altura da base (DAB). Da mesma forma, é necessário que inicialmente se tenha acesso aos DAP's e os DAB's medidos a campo. A partir desses valores, pode-se então ajustar modelos de regressão para estimar os DAP's 
das árvores colhidas em função dos DAB's remanescentes, reconstituindo-se, assim, a biomassa e/ou o volume utilizando-se equações alométricas desenvolvidas para o local de estudo (LIMA, 2016).

Desta forma, considerando a importância das mensurações a partir do DAB, o presente estudo teve como objetivo modelar variáveis dendrométricas de árvores individuais de Pinus taeda L. em função do diâmetro medido a 0,1 e 0,3 metros acima do nível do solo.

\section{MATERIAL E MÉTODOS}

\section{Área de estudo}

Árvores de Pinus taeda L. crescendo livre de competição foram amostradas em uma área rural no município de Capão Alto (SC), com coordenadas de Latitude: $27^{\circ} 56^{\prime}$ S e Longitude: $50^{\circ} 30^{\prime} \mathrm{O}$. O clima, de acordo com a classificação de Köppen, é subtropical úmido, sem estação seca e com verão temperado (Cfb). A altitude média em relação ao nível médio do mar é de $950,2 \mathrm{~m}$, a temperatura anual média (TAM) é de $15,4{ }^{\circ} \mathrm{C}$ e a precipitação acumulada anual média (PAM) é de $1735 \mathrm{~mm}$ (ALVARES et al., 2013).

\section{Coleta de dados}

Um total de 20 árvores de Pinus taeda L. foram abatidas e registrados os seus diâmetros com casca das posições $0,1,0,3$ e 1,3 m, e a partir desta posição, de metro em metro, até alcançar a altura total. As variáveis diâmetro à altura do peito (DAP) e altura total (h) foram medidas. Para a obtenção do volume rigoroso da árvore foi usado a fórmula de Smalian.

\section{Modelo utilizado para descrever as variáveis dendrométricas}

A descrição do comportamento das variáveis dendrométricas em função do diâmetro medido a $0,1\left(\mathrm{~d}_{0,1}\right)$ e $0,3\left(\mathrm{~d}_{0,3}\right)$ metros acima do nível do solo foram avaliadas de acordo com o modelo linear simples (1).

$$
y=\beta_{0}+\beta_{1} \cdot x_{1}+\varepsilon
$$

Onde: $\mathrm{y}=$ [diâmetro à altura do peito (DAP), em $\mathrm{cm}$; altura total $(\mathrm{h})$, em m; volume com casca $\left.(v), m^{3}\right] ; x_{1}=$ diâmetro medido a $0,1\left(d_{0,1}\right)$ ou $0,3\left(d_{0,3}\right)$ metros acima do nível do solo; $\varepsilon=$ erro residual.

\section{Análise dos dados}

Os modelos de regressão foram ajustados utilizando técnicas de Modelos Lineares Generalizados (MLG) (FOX, 2015) no software R (R DEVELOPMENT CORE TEAM, 2013). Para o ajuste considerou-se a distribuição aleatória contínua gamma e a função de ligação logarítmica (In ). O teste de Wald foi utilizado como teste de hipótese para observar a significância dos coeficientes da equação.

A verificação da performance dos modelos foi baseada no cálculo do coeficiente de determinação $\left(R^{2}\right)$, o erro padrão da estimativa em porcentagem (Syx\%), valor do critério de informação de Akaike (AIC), do critério de informação Bayesiano $(\mathrm{BIC})$ e a análise gráfica dos resíduos. A manipulação de dados foi realizada com a planilha eletrônica Excel Versão 2016. 


\section{RESULTADOS}

A amplitude de distribuição diamétrica das árvores de Pinus taeda L. foi de 27,4 a $58,8 \mathrm{~cm}$ (Tabela 1). A altura média foi de $28,8 \mathrm{~m}$, com desvio-padrão de $\pm 4,8$ $\mathrm{m}$. A variação da altura das árvores é reflexo dos diferentes sítios amostrados e de sua capacidade de crescimento na condição que estavam inseridas, pois, as árvores estudadas cresciam livre de competição com as outras árvores por espaço e luz.

TABELA 1 - Resumo estatístico das características dendrométricas de árvores de Pinus taeda.

\begin{tabular}{cccccc}
\hline Variável & Mínimo & Média & Mediana & Máximo & Desvio-Padrão \\
\hline DAP & 27,4 & 41,2 & 39,5 & 58,8 & 10,1 \\
$\mathrm{~d}_{0,1}$ & 32,5 & 52,3 & 50,8 & 94,1 & 16,2 \\
$\mathrm{~d}_{0,3}$ & 30,9 & 47,3 & 45,4 & 72,3 & 12,2 \\
$\mathrm{~h}$ & 21,1 & 28,8 & 28,3 & 37,0 & 4,8 \\
$\mathrm{v}$ & 0,6355 & 2,1114 & 1,6563 & 4,5770 & 1,2269 \\
\hline
\end{tabular}

Em que: $\mathrm{DAP}=$ diâmetro à altura do peito, em $\mathrm{cm}$; $\mathrm{d}_{0,1}=$ diâmetro medido a 0,1 metros acima do nível do solo; $\mathrm{d}_{0,3}=$ diâmetro medido a 0,3 metros acima do nível do solo; $\mathrm{h}=$ altura total, em $\mathrm{m} ; \mathrm{v}=$ volume com casca, em $\mathrm{m}^{3}$.

Com o ajuste do modelo 1 , foi possível comparar e selecionar quais as variáveis independentes $\left(d_{0,1}\right.$ e $\left.d_{0,3}\right)$ deveriam ser utilizadas na estimativa dos atributos dendrométricos analisados (Tabela 2). Os resultados indicaram superioridade das equações ajustadas em função do $\mathrm{d}_{0,3}$, com os melhores valores para os critérios estatísticos avaliados.

TABELA 2 - Coeficientes de regressão e critérios estatísticos usados para avaliar a performance das equações ajustadas de árvores de Pinus taeda.

\begin{tabular}{cccccccc}
\hline $\mathbf{y}$ & $\mathbf{x}_{\mathbf{1}}$ & $\boldsymbol{\beta}_{\mathbf{0}}$ & $\boldsymbol{\beta}_{\mathbf{1}}$ & $\mathbf{R}^{\mathbf{2}}$ & $\mathbf{S y x} \%$ & $\mathbf{A I C}$ & $\mathbf{B I C}$ \\
\hline DAP & & 2,9594 & 0,0141 & 0,72 & 13,4 & 119 & 122 \\
$\mathrm{~h}$ & $\mathrm{~d}_{0,1}$ & 2,9469 & 0,0078 & 0,56 & 11,4 & 108 & 111 \\
$\mathrm{v}$ & & $-1,3091$ & 0,0366 & 0,20 & 53,6 & 33 & 36 \\
\hline DAP & & 2,7480 & 0,0199 & 0,95 & 5,4 & 86 & 88 \\
$\mathrm{~h}$ & $\mathrm{~d}_{0,3}$ & 2,8255 & 0,0111 & 0,69 & 9,5 & 103 & 106 \\
$\mathrm{v}$ & & $-1,7943$ & 0,0502 & 0,87 & 21,9 & 10 & 13 \\
\hline
\end{tabular}

É notável que o ajuste do modelo 1 , com a utilização da variável independente $\mathrm{d}_{0,3}$, é bastante eficiente para estimar as variáveis de interesse da área em estudo, mostrando que é possível estimar a altura e o volume, através de uma variável independente que não seja o DAP com precisão. Assim, com a utilização do $\mathrm{d}_{0,3}$ é possível estimar o DAP das árvores com bons resultados estatísticos.

O uso dessa equação torna possível aplicações relacionadas à reconstituição das características dendrométricas de florestas, sendo uma ferramenta confiável para realização de perícias, falta de dados, erros amostrais e não amostrais em inventários, aplicando o conceito de engenharia reversa, isto é, partindo da pós colheita para estimativa da estrutura inicial da floresta.

A tendência das linhas médias de regressão estimadas para as variáveis DAP, $h$ e $v$ em função do $d_{0,3}$ são apresentadas na Figura 1. As diferentes tendências mostram as mudanças de tamanho e forma que ocorrem nas árvores 
quando em locais sem concorrência com outras árvores por recursos bióticos a abióticos.

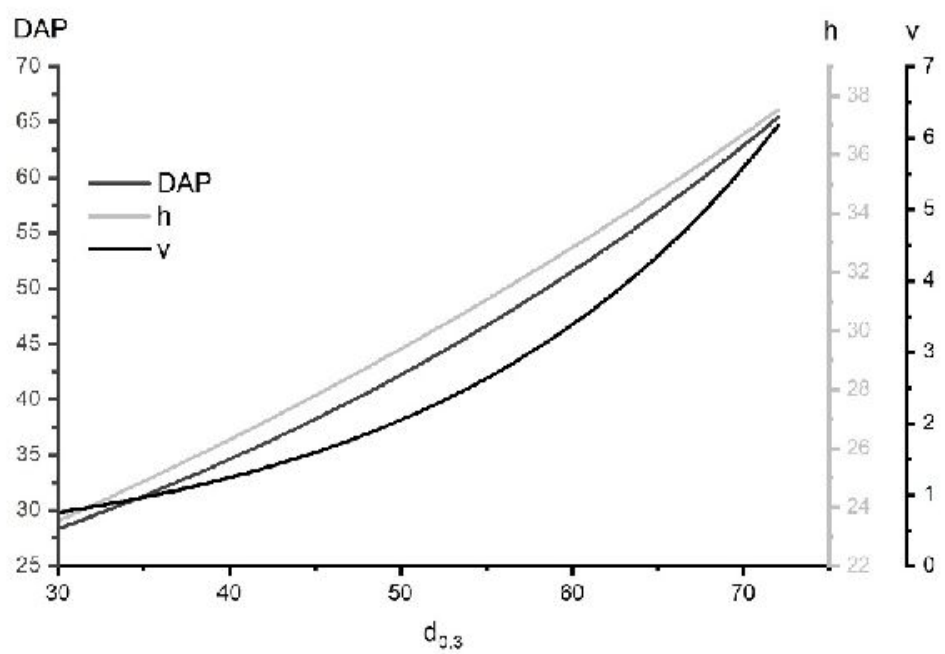

FIGURA 1 - Relação entre DAP, h e v em função do $d_{0,3}$.

\section{DISCUSSÃO}

Rivas et al. (2007) desenvolveram equações para estimar DAP e volume de árvores a partir do diâmetro do toco sem casca para as principais espécies de pinus na região florestal de El Salto, Durango (México). Esses autores consideraram o diâmetro do toco sem casca, pois geralmente a casca não é utilizada com um fim comercial, portanto, estimar o diâmetro sem ela resulta em mais precisão. Similar ao presente estudo, os autores concluíram que equações de regressão podem ser utilizadas para estimar os atributos da árvore e, quando modeladas equações para diferentes espécies, sugerem testar diferentes equações, permitindo selecionar melhores equações para cada espécie, auxiliando pesquisadores que perderam árvores em seus ensaios experimentais, por corte, entre outras utilizações.

Özçelík et al. (2010) realizaram um estudo com objetivo da predição do DAP e volume da árvore em função do diâmetro de toco com casca, para três espécies de árvores economicamente importantes na região florestal de Bucak, Turquia. Para estimar o DAP, foram ajustados três modelos de regressão (linear, quadrático e exponencial), e para estimar o volume foram ajustados dois modelos de regressão (exponencial e quadrático). Os autores constataram que o modelo linear simples estima o DAP com precisão, já o volume total da árvore é estimado com precisão quando utilizada equação exponencial. Além disso, os autores também afirmaram a necessidade de ajustes específicos para cada espécie.

Lima (2016), avaliou a reconstrução da composição, estrutura e biomassa arbustiva-arbórea a partir de cepas em uma área de Caatinga sob Plano de Manejo Florestal Sustentável (PMFS). Os dados foram coletados após dois anos do corte, portanto a vegetação já estava em estágio de rebrota ou regeneração. O autor concluiu que a reconstituição da biomassa existente na área antes do corte foi possível por meio da estimativa do DAP das cepas e de uma equação já ajustada de biomassa para a Caatinga, tomando como base o DAP. Este tipo de modelo tornase uma ferramenta eficaz para os órgãos ambientais autuarem possíveis infrações no que diz respeito a supressão de vegetação ilegal.

Rivas et al. (2007) mencionam que as equações dendrométricas em função das dimensões do toco representam novas e úteis ferramentas do manejo florestal 
para os silvicultores, proprietários florestais e autoridades fiscais, elucidando a importância do presente estudo para $P$. taeda, considerando a relevância econômica da espécie.

\section{CONCLUSÕES}

As equações ajustadas permitem descrever com acurácia e precisão as variáveis dendrométricas em função do $\mathrm{d}_{0,3}$. Essas equações podem auxiliar na quantificação dos atributos da árvore apenas com base nas dimensões do toco.

Com as equações obtidas é possível realizar a reconstituição da estrutura do conjunto de árvores após do corte.

\section{REFERÊNCIAS}

ALVARES, C. A.; STAPE, J. L.; SENTELHAS, P. C.; GONÇALVES, J. L. M.; SPAROVEK, G. Köppen's climate classification map for Brazil. Meteorologische Zeitschrift, v. 22, p. 711-728, 2013.

ARANDA, U. D.; ANTA, M. B.; DORADO, F. C.; MURIAS, M. B. Estimación del diámetro normal y del volumen del tronco a partir de las dimensiones del tocón para seis especies forestales comerciales de Galicia. Investigación Agraria: Sistemas y Recuros Forestales, v. 12, n. 2, p.131-139, 2003. Disponível em: $<$ https://www.researchgate.net/publication/28063129_Estimacion_del_diametro_nor mal_y_del_volumen_del_tronco_a_partir_de_las_dimensiones_del_tocon_para_seis _especies_forestales_comerciales_de_Galicia>.

ERCANLI, I.; GUNLU, A.; BASKENT, E. Z. Mixed effect models for predicting breast height diameter from stump diameter of Oriental beech in Göldag. Scientia Agricola, v. $72, \quad$ n. 3, p. 245-251, 2015. Disponível em: $<$ http://www.scielo.br/scielo.php?script=sci_arttext\&pid=S010390162015000300245\& Ing=en\&tlng=en>. doi: http://dx.doi.org/10.1590/0103-9016-2014-0225.

FOX, J. Applied Regression Analysis and Generalized Linear Models. 3ํe., Sage Publications Inc. 2015. 816p.

IBA. Indústria Brasileira de Árvores. Relatório IBÁ - 2017. O setor brasileiro de árvores plantadas referente ao ano de 2017. Disponível em: <https://iba.org/images/shared/Biblioteca/IBA_RelatorioAnual2017.pdf>.

IBGE. Produção da Extração Vegetal e da Silvicultura. 2017. Disponível em: $<$ https://agenciadenoticias.ibge.gov.br/agencia-sala-de-imprensa/2013-agencia-denoticias/releases/22620-pevs-2017-producao-da-silvicultura-e-da-extracao-vegetalchega-a-r-19-1-bilhoes-e-cresce-3-4-em-relacao-a-2016>.

LIMA, T. L. Reconstituição da composição, estrutura e biomassa arbustivaarbórea a partir de cepas em caatinga sob manejo florestal. 2016. 75 f. Dissertação (Programa de Pós-Graduação em Ciências Florestais) - Universidade Federal Rural de Pernambuco, Recife. Disponível em: <http://www.tede2.ufrpe.br:8080/tede2/handle/tede2/7407>.

ÖZÇELÍK, R.; BROOKS, J. R.; DIAMANTOPOULOU, M. J.; WIANT JR, H. V. Estimating breast height diameter and volume from stump diameter for three 
economically important species in Turkey. Scandinavian Journal of Forest Research, v. 25, n. 1, p.32-45, 2010. Disponível em: <https://www.tandfonline.com/doi/abs/10.1080/02827580903280053>. doi: http://dx.doi.org/10.1080/02827580903280053.

PETER, D. H.; CONSTANCE, A. H. Reconstructed old-growth forest stand structure and composition of two stands on the Olympic Peninsula, Washington state. DIANE Publishing, 2010.

R Core Team. R: A language and environment for statistical computin. R Foundation for Statistical Computing, Vienna, Austria, 2013. Disponível em: <https://www.Rproject.org/>.

RIVAS, J. J. C.; ANTA, M. B.; CALDERÓN, O. A. A.; ARANDA, U. D.. Use of stump diameter to estimate diameter at breast height and tree volume for major pine species in El Salto, Durango (Mexico). Forestry, v. 80, n. 1, p.29-40, 2007. Disponível em: <https://academic.oup.com/forestry/article/80/1/29/634526>. doi: http://dx.doi.org/10.1093/forestry/cpl048. 